\title{
FP1723 PHOTODYNAMIC THERAPY IN SKIN ULCER CLOSURE
}

\author{
S. Motta ${ }^{1}$, D. Kanah ${ }^{1}$, M. Monti ${ }^{1}$ \\ ${ }^{1}$ Università degli Studi di Milano, Istituto Clinico Humanitas, Rozzano, Italy
}

Wound healing is the process of restoring integrity to traumatised tissue, and involves the biological principles of tissue response to injury with all of its associated inflammatory, immune, biochemical, cell biology and signal transduction mechanisms. Photodynamic therapy with 5-aminolevulinic acid (ALA-PDT) is widely used in dermatology to destroy malignant skin tumours and induce remission in some inflammatory disorders, and some recently published data indicate that skin venous ulcers can also be targeted. Our Department has a specific unit dedicated to healing skin ulcers, in which we have been using multisession ALA-PDT for three years to treat ulcers of all pathogenetic causes. In order to establish the role of ALA-PDT in wound healing, we experimentally evaluated the efficiency of red light vs red light $+A L A$, the antibacterial activity of ALA-PDT, and the activity of ALA-PDT vs a standard dressing in treating 13 cases of chronic venous ulcers, four of autoimmune ulcers, three of hypertensive arterial ulcers, and two cases of ulcerated radiodermitis. All of the selected patients had multiple and/or large ulcers (area $>20 \mathrm{~cm} 2$ ). After obtaining the patients' formal informed consent, multiple ulcers were divided into groups and treated with:1) our standard dressing alone; 2) our standard dressing and weekly ALA-PDT; and 3) our standard dressing and weekly red light exposure. The ALA-PDT treatment consisted of the application of $10 \%$ ALA in polyethylenglycol (PEG) ointment in occlusion for 24 hours, followed by exposure to diode red light at $630 \mathrm{~nm}$, irradiance $160 \mathrm{~mW}$ at $50 \mathrm{~mm}$, for eight minutes delivering $75 \mathrm{~J} / \mathrm{cm} 2$. Microbiological samples were collected from all of the ulcers for the primary isolation of gram-negative bacilli, gram-positive cocci and mycetes before the application of ALA or dressing, after 24 hours' ALA or dressing occlusion, and after exposure to red light.

On the basis of our 3-year experience, we can say that multisession ALA-PDT heals all of the treated ulcers, and in half the time required by the standard dressing in the same patient; red light exposure alone does not promote wound healing; and ALAPDT does not have any antibacterial activity. These in vivo results demonstrate the favourable activity of ALA-PDT in wound healing, although the underlying biological mechanisms are still unclear.

References: Br J Dermatol. 2003, 149 (Suppl. 64), 87-93

Biol Cell. 2005 March:97(3):173-183

Br J Dermatol. 2004 Sep;151(3):616-22.

Photochem Photobiol Sci. 2002 Jan;1(1):79-80

Photodiagn and Photodynam Therapy,2005,2,4, 283-298

J Dermatolog Treat. 2006;17(4):222-3.

Acta Derm Venereol. 2007;87(4):325-9.

Photochem. Photobiol. Sci., 2007, 6, 1150-1151 Photodynamic therapy - a promising treatment option for autoimmune skin ulcers:a case report. S.Motta, M.Monti 